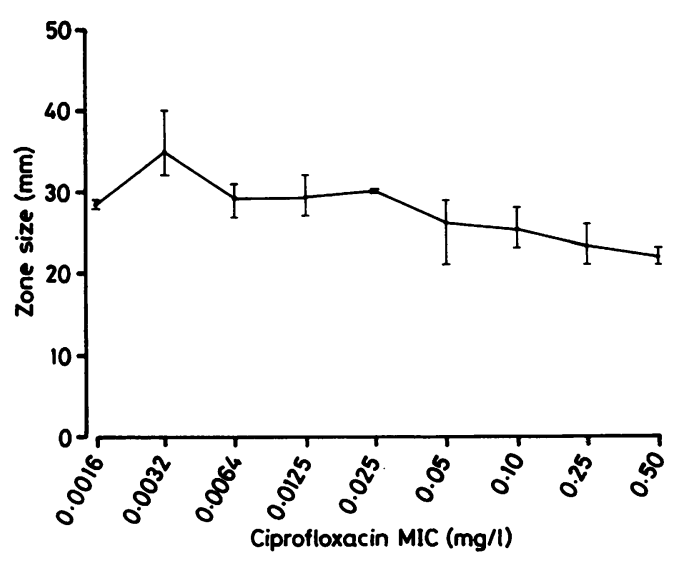

Figure 1 Inhibition zone size around a $5 \mu \mathrm{g}$ ciprofloxacin disc for isolates with the indicated ciprofloxacin MIC. Bars indicate the range of zone size found and the line indicates the mean zone size.

\section{Discussion}

We have shown that the $30 \mu \mathrm{g}$ nalidixic acid disc can be used to provide a good screening test for ciprofloxacin resistance in $N$ gonorrhoeae. The results suggest that laboratories which serve a genitourinary medicine clinic where ciprofloxacin is used could adopt this test to detect those strains for which this drug is not appropriate.

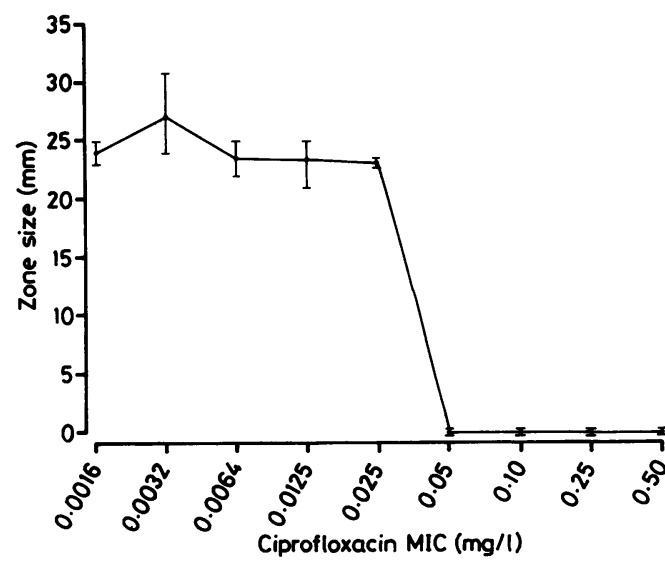

Figure 2 Inhibition zone size around a $30 \mu \mathrm{g}$ nalidixic acid disc for isolates with the indicated ciprofloxacin MIC. Bars indicate the range of zone size found and the line indicates the mean zone size.

1 Lefevre JC, Tempesta MC, Gaubert E, Lareng MB. Invitro activity of six quinolone derivatives against Neisseria gonorrhoeae. Chemotherapy 1988;34:315-7.

2 Klingeren van B, Dessens-Kroon M, Verheuvel M. In-vitro activity of quinolones against penicillinase-producing and non-penicillinase-producing gonococci. Chemioterapia 1985;4(suppl 2):464-5.

3 Melby K, Faegri A. The in-vitro activity of norfloxacin, ofloxacin and ciprofloxacin and other antibiotics in current use against Neisseria gonorrhoeae. APMIS 1989 97:347-50.

4 Gransden WR, Warren CA, Phillips I, Hodges M, Barlow $D$. Decreased susceptibility of Neisseria gonorrhoeae to ciprofloxacin. Lancet 1990;335:51.

5 Jephcott AE, Turner A. Ciprofloxacin resistance in gonococci. Lancet 1990;335:165.

\title{
Examination of spinal cord in diseases of the craniocervical junction and high cervical spine
}

\author{
J F Geddes, A G Gonzalez
}

\author{
Department of \\ Neuropathology, \\ National Hospital for \\ Neurology and \\ Neurosurgery, Maida \\ Vale, London W9 1TL \\ J F Geddes \\ National Hospital for \\ Neurology and \\ Neurosurgery, \\ Queen's Square, \\ London WC1 \\ A G Gonzalez \\ Correspondence to: \\ Dr J F Geddes \\ Accepted for publication \\ 16 August 1990
}

\begin{abstract}
A simple necropsy technique for the removal of the craniocervical junction was devised: a relatively small specimen comprising part of the clivus, the foramen magnum, and cervical vertebral canal is removed in one piece with the medulla and spinal cord inside, and examined systematically after fixation. This method, used in a series of patients with chronic craniocervical instability, allows both good clinicopathological correlations to be made and histological changes in the lower medulla or upper cervical cord segments to be related to sites of extrinsic compression.
\end{abstract}

Chronic medullospinal or high cervical cord compression produces brainstem symptoms and upper cervical myelopathy with long tract signs. Clinicopathological correlations are not often described, because it is relatively difficult to examine the craniocervical junction. Some pathologists remove the entire bony column with the spinal cord inside, but may not include the foramen magnum. This is also a time consuming procedure, creating problems in reconstruction for the mortuary technician. We performed necropsies on 15 patients with atlantoaxial and subaxial dislocation, 13 of whom had had transoral surgery for decompression of the cord, in almost 


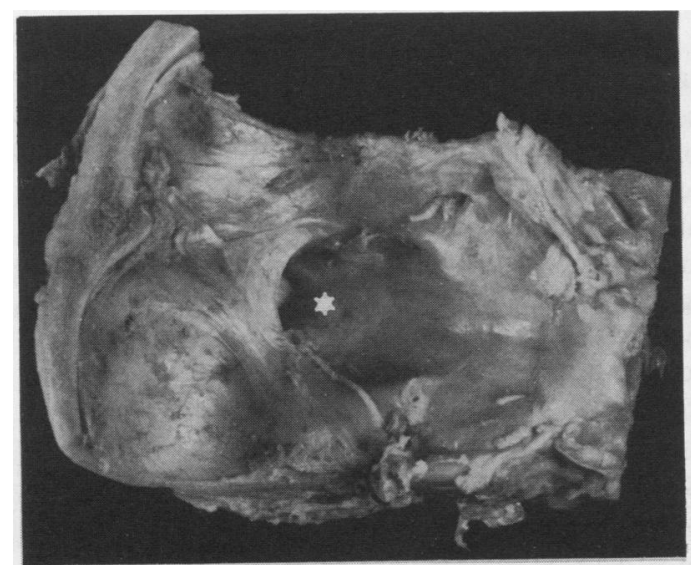

Figure 1 The bony specimen, removed from the body and viewed from above. The cervical cord can just be seen through the foramen magnum, and the canal is much narrowed by posterior displacement of the odontoid ( $\left.{ }^{\star}\right)$.

all cases combined with a stabilisation procedure. The underlying pathologies included: rheumatoid arthritis $(n=8)$, vertebral body lymphoma ( $\mathrm{n}=1)$, Down's syndrome ( $\mathrm{n}=$ $2)$, cervical spondylosis $(n=1)$, osteogenesis imperfecta $(n=1)$, and two cases in which cord compression was caused by degenerate ligaments forming a mass behind $\mathrm{C} 2$.

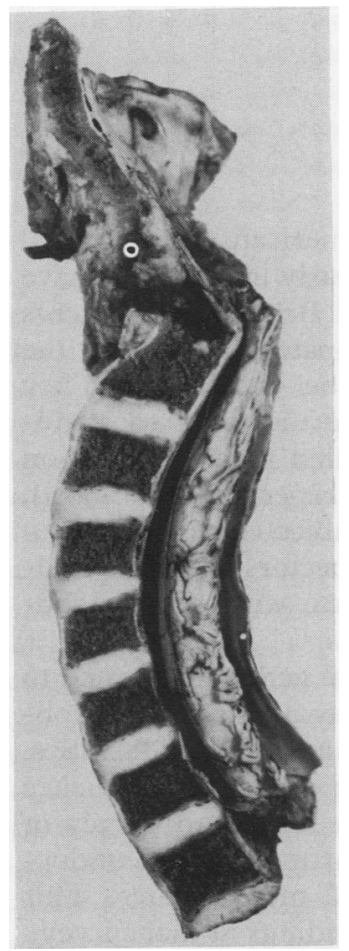

Figure 2 A hemisected spine with the cord replaced. This shows the retraction of the cord that has occurred during fixation (seen also to a lesser extent in fig 1); in subsequent necropsies, higher cuts were made higher cuts were made removal of the brain. pontomedullary junction. If the usual transverse cut just below the medulla is made in the presence of severe narrowing of the upper

\section{thods}

body, but because the scalp line must be exposure of the vertebral laminae, three anterior neck incisions result.

of the neck in the midline, bringing the cut up over the scalp as a sagittal incision. This should more difficult in the presence of oedema than with a coronal incision. With this method, either a U-shaped or a midline cut can be used for opening the neck from the front.

opening the neck from the front. spinal canal, the cut end of the cord tends to retract slightly into the site of constriction during fixation, making it difficult to take proper blocks of the cord at the level of the pathology.

After removal of the brain the degree of movement, particularly of the odontoid in relation to the foramen magnum, can be assessed by careful palpation with the head flexed. An oscillating saw fitted with a wedge-shaped blade is then used to cut a block through the base of the skull, about $2 \mathrm{~cm}$ each side of the foramen magnum (fig 1). The transverse anterior saw cut may be made at a greater distance, to include all of the clivus. When surgical fixation has been performed, the posterior cut across the occiput is made to include any wires present in the skull.

Inside the body, a disc space is chosen well below the level of bony pathology (C7-T1 is suitable for high cervical compression), and the column is cut through with a body knife. The origin of the vertebral arteries should be included in the specimen (usually C6-7). The knife is then used to dissect the deep muscles of the neck off the cervical column, and the specimen should come out without difficulty. Note that in the presence of craniocervical instability, it is easy to damage the cord during this procedure if the skull base has not been completely cut through and if any force is used to free it. The whole specimen once removed is fixed in $10 \%$ formalin. (We find that $25 \%$ formalin, used in some centres for fixing the brain, tends to produce a peripheral staining artefact that is particularly pronounced in silver axon preparations.) Finally, the lower part of the cord can be removed in the usual way if required.

Reconstruction of the neck is usually achieved with a wooden strut, but we often use instead the length of vertebral bodies removed when the thoracolumbar cord is taken, and even padding with cotton wool alone may sometimes be sufficient.

After fixation, the appearance and dimensions of the foramen magnum are recorded, and the specimen is further cleaned of soft tissue. Any wires that have been inserted as part of a stabilisation procedure can be removed carefully with pliers. The spinal laminae are then sawn through on each side, the cuts extending up through the occipital bone. This exposes the dura, which is opened in the midline with a pair of scissors. The cord is gently lifted beginning at the caudal end, and anterior and posterior nerve roots cut through in succession. Once the cord is removed, the bony specimen is sawn through in the sagittal plane.

The cord can then be replaced in the hemispine, and it is easy to relate individual cord segments to sites of compression (fig 2). Conventional transverse blocks of cord at different segments yield more information for clinicopathological correlation than longitudinal sections of a cord that has been bisected with the vertebral spine. When facilities exist, finedetail $x$-ray pictures of a bony block, followed by decalcification, and paraffin wax embedding, may be useful. 


\section{Discussion}

This technique is not merely an academic exercise, of use only in centres specialising in high cervical surgery. Spontaneous atlantoaxial dislocation in rheumatoid arthritis and ankylosing spondylitis is relatively common, particularly in patients admitted to hospital for their disease, in whom figures of $18 \%,{ }^{1} 28 \%,{ }^{2}$ and $40 \%{ }^{3}$ have been reported. Sudden death after anaesthesia may occur if patients with unrecognised craniocervical instability undergo surgery, and the cause of death may be missed at necropsy if the medullospinal junction is not examined. The method that we have described is not difficult, if an electric saw is available, and takes very little additional time at necropsy. Provided a reasonable length of medulla/cord is left rostral to the site of constriction, the correlation between pathology, symptoms, and signs may be made with relative success.

We are grateful to Dr J T Hughes for help and encouragement in the prepartion of some of the specimens; to Drs R O Barnard the preparation of some of the specimens; to Drs $R$ a Barnard and Steve Durr for photographs.

1 Sharp J, Purser DW. Spontaneous atlanto-axial dislocation in ankylosing spondylitis and rheumatoid arthritis. Ann in ankylosing spondylitis and

Rheum Dis 1961;20:47-77.
2 Eulderink R, Meijers KA. Pathology of the cervical spine in rheumatoid arthritis. J Pathol 1976;120:91-109.

3 Serre H, Simon L. Atlanto-axial dislocation in rheumatoid arthritis. Rheumatism 1966;22:53-8.

\section{Royal College of Pathologists' accreditation pilot study: a year later}

The College Audit Steering Committee

Members of the committee: Dr G W Pennington (chairman), Professor C L Berry, Dr D Burnett, Dr J C Coleman, Mr B Edwards, Mrs M Fox, Professor I Lauder, Dr J S Lilleyman, Dr B Murphy, Dr E Walton

Correspondence to:

Dr J S Lilleyman, The Children's Hospital, Western Bank, Sheffield S10 2TH.

Accepted for publication 31 July 1990

\begin{abstract}
Twenty one heads of departments who participated in the Royal College of Pathologists' pilot study of laboratory accreditation were questioned a year after the event to discover if the process had produced any lasting effect. All but one responded. Eighteen $(90 \%)$ felt that there had been lasting and tangible benefit from their inspection. Of 10 departments with deficiencies relating to resources (and where no plans to upgrade existed before the study), five $(50 \%)$ had obtained improvements as a direct result of the inspector's report. Overall, $16(80 \%)$ of respondents were in support of the development of an accreditation scheme of the type attempted, and none were opposed.

The envisaged scheme seems to have the potential to produce lasting improvements in service and commands the support of the departments involved so far.
\end{abstract}

In July 198921 diagnostic pathology departments from six assorted hospitals agreed to participate in a pilot accreditation study based on the College of American Pathologists' format. The event and early impressions have already been described. ${ }^{1}$ Briefly, departments volunteering to participate applied to the scheme organisers to be accredited. They received a book defining the required standards of performance, filled in an application form listing details of repertoire, workload, and staffing, and then subjected themselves to on-site visits from inspectors who methodically checked compliance with those standards using long checklists.

Technical performance generally seemed to be satisfactory, but several important problems were found in organisation or facilities. To discover what lasting effect the experience might have produced, a follow up survey of heads of volunteer departments was conducted in June of 1990, 12 months later. This report summarises the findings of that survey.

\section{Methods}

Each of the 21 heads was asked the following questions:

1 Was there any tangible benefit from your inspection? If so, what?

2 Have there been any material changes in 